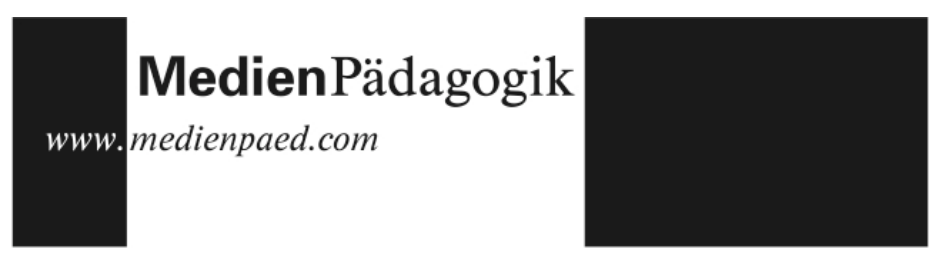

Rezensionen

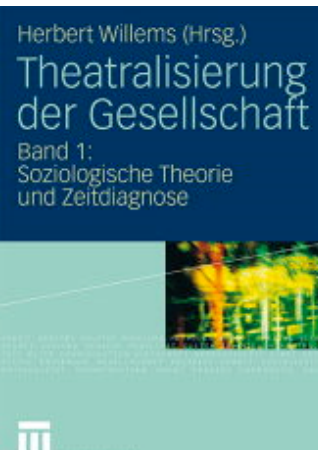

Herbert Willems (Hg.)

Theatralisierung der Gesellschaft.

Soziologische Theorie und Zeitdiagnose. Bd. 1

Wiesbaden: VS Verlag für Sozialwissenschaften, 2009. 631 Seiten

ISBN-13 978-3-531-14922-6

$€$ 49.90; CHF 85.00

Medientheatralität und Medientheatralisierung. Bd. 2

Wiesbaden: VS Verlag, 2009. 467 Seiten

ISBN-13 978-3-531-14921-9

$€$ 49.90; CHF 85.00

\title{
Die Aufführung von Sozialität - \\ Theatermetaphorik als erkenntniskonstitutives Modell sozialen Wandels
}

Die soziologische Diagnose umfassender soziokultureller Veränderungsprozesse hängt eng mit der Entstehung des Faches selbst zusammen. Solche Diagnosen wie etwa jene einer entstandenen Wissens- oder Eventgesellschaft sind durch die Nachbardisziplinen wie die Erziehungswissenschaft und hier die (Medien-)Bildungsforschung als auch in den vergangenen Jahren vor allem durch die Medienpädagogik für eigene Forschungsperspektiven immer wieder aufgegriffen worden.

\section{Problemhorizont und Aufbau}

Herbert Willems, der Herausgeber der beiden Sammelbände Theatralisierung der Gesellschaft, vereinigt Beiträge aus verschiedenen Disziplinen mit sehr unterschiedlichen Forschungsinteressen, die einen integrativen Blickwinkel auf soziokulturelle Phänomene bieten sollen (Bd.1,13). Diese Integrationsleistung soll über den «analytisch spezifisch leistungsfähigen, aber auch konzeptuell theoretisch integrativen und anschlussfähigen Schlüsselund Leitbegriff» der Theatralisierung (14) hergestellt werden. Das zu Grunde liegende Theatralitätskonzept fokussiert in Anlehnung an die Theater- und Kulturwissenschaftlerin Erika Fischer-Lichte auf «Performance» (körperliche Darstellung vor Publikum), «Inszenierung» (Produktionsprozess der Performance), «Korporalität und Wahrnehmung» (Betrachterperspektive) (vgl. ausführlich: 78ff). Der verfolgte Ansatz geht zugleich über die historische und interaktionszentrierte Begrenzung jenes Theatralitätskonzeptes hinaus, so der Herausgeber, und soll im Sinne Goffmans Formen von Theatralität im Alltag und kulturhistorischen Zusammenhang in den Blick rücken (vgl. 15f, 59). 
Die Einzelbeiträge beziehen sich also neben jenem Rekurs auf FischerLichte auf weitere Interpretationsansätze, um die "sozio-kulturelle Entwicklungslogik und Entwicklungstendenz der Gegenwartsgesellschaft» (17) als gerichteten Wandel anhand heterogener und gegenläufiger Prozesse der (Ent-)Theatralisierung (23) zu bezeichnen.

Die Bände sind an ein soziologisch interessiertes breites Fachpublikum gerichtet, dürften aber auch außerhalb akademischer Rezeption ein Publikum finden. Der erste Band gliedert sich in eine Einleitung vom Herausgeber, die eine durch die Autoren erstellte Zusammenfassung der Einzelbeiträge enthält, und drei weitere Kapitel. Diese beschäftigen sich mit (Ent)Theatralisierungsphänomenen auf «verschiedenen gesellschaftlichen Ordnungsebenen» (58) in den Bereichen «Alltägliche und außeralltägliche Lebenswelten» (9 Beiträge), «Spezielle (Gruppen-)Kulturen» (5 Beiträge) und "Soziale Felder» (13 Beiträge). Der zweite Band fokussiert (Medien)Theatralität mit Schwerpunkt auf den Massenmedien und dem Internet, insofern davon ausgegangen wird, dass Medien eine zunehmend komplexe und eigenständige Bedeutung für Prozesse der (Ent-)Theatralisierung haben (vgl. 58).

Theatralität wird von Willems als figurationssoziologisches Konzept verstanden, dass neben den benannten Referenzen auch an die Arbeiten der Soziologen Norbert Elias und Pierre Bourdieu anknüpft (vgl. 75ff). Die Metaphorik des Theaters soll dabei in Goffman'scher Manier als analytisches, erkenntniskonstitutives Modell die Realität des Theatralischen hinter der vertrauten Oberfläche des Sozialen sichtbar machen, wobei dessen Begrenzung mit reflektiert wird (vgl. 77). Die figurationssoziologische Perspektiverweiterung durch Elias und Bourdieu ist in der kulturhistorischen Kontextualisierung zu sehen (vgl. 102ff), die sowohl auf die strukturierende Bedeutung von sozialisatorischen Figurationen (etwa soziale habitusgenerierende Felder) als auch Handlungsspielräume der Akteure blickt. In (und zuweilen auch außerhalb) dieser Rahmung sind in zwei Bänden unterschiedliche Einzelbeiträge situiert.

\section{Soziologische Theorie und Zeitdiagnose (Band 1)}

Willems konturiert in seinem Beitrag das Konzept des (Lebens-)Stils und seinen analytischen Gehalt innerhalb des Theatralitätsmodells. Er konstatiert eine universelle Theatralisierungstendenz im Lebensstil Jugendlichkeit ins- 
besondere am Beispiel massenmedialer «Stil-Bühnen» und hier der Werbung, wobei der gegenwärtige generalisierte Jugendlichkeitskomplex einen vielfältigen Stilismus hervorbringe und die Aufmerksamkeit auf die Oberfläche des Körpers lenke (vgl. 132). Die kulturelle «Verjugendlichung» von Kindheit und höherem Erwachsenenalter ist neben jener Körperbetonung mit einer kulturellen «Verspielung» verknüpft (vgl. 133).

Auch Klaus R. Schroeter diagnostiziert ein breit wirksames Jugendlichkeitsideal im Alter. In seinen Ausführungen über «Korporales Kapital in der Lebensphase Alter» beschreibt er korporale Kompetenzen im Kontext des Doing Age, die auf den «verbliebenen Abstand des 'nicht mehr' (Könnens, Dazugehörens u.s.w.)" verweisen (168). Die Darstellung von Alter ereignet sich habituell oder inszeniert, beispielsweise in der Betonung von Erfahrungswissen (vgl. 169). Alter unterliegt in seiner Theatralität den gesellschaftlichen, auf die Idealität von Jugendlichkeit bezogenen Imperativen Fitness und Wellness mit vielfältigen körpertechnologischen Ausdrucks- und Erscheinungsformen und (re-)produziert eine Aufwertung des produktiven und erfolgreichen Alterns (vgl. 177).

In Rekurs auf Goffman veranschaulicht Ernst von Kardorff die (ReProduktion von Stigmata im Kontext reflexiv-moderner Rahmenverschiebungen und -vermischungen. Funktionalistisch betrachtet er die durch die Forderung nach Teilhabe und Selbstbestimmung entgrenzte Pflege (vgl. 150ff), welche vermehrt emotionale Aushandlungsprozesse hinsichtlich sozialer (Ent-)Stigmatisierung erforderlich macht (vgl. 155). Modernisierungsund professionstheoretisch als auch unter Einbezug emotionssoziologischer Perspektiven insistiert er auf den Stellenwert aktiven Stigmamanagements etwa mit theatralisierten Kommunikationsmitteln wie dem dramaturgischen Körper für die (narrative) «Neurahmung der eigenen Biografie um das kritische Lebensereignis herum (reframing)» (156).

\section{Theatralität veränderter Privatheit}

Das Verhältnis von Theatralität und Intimität wird auch in den Beiträgen von Günter Burkart, Karl Lenz und Thomas Schwietring behandelt. Burkart sieht in der Inszenierung des mobilen Selbst durch die raum-zeitliche Entbindung von Kommunikation die Verwischung von privaten und öffentlichen Sphären vor dem Hintergrund veränderter Intimität und bestehenden Intimitätsregeln und eruiert die Ambivalenz von Flexibilisierung in diesem Zusammenhang (vgl. 208ff). Lenz konstatiert einen durch Individualisierungsprozesse ent- 
standenen "sozialen Zwang der Theatralität im Beziehungsaufbau»(241), welcher eine Ambivalenz exklusiver und zugleich (massenmedial) konfektionierter Intimität erzeugt (vgl. 241ff). Schwietring beschreibt Intimität als Ergebnis kulturspezifischer «Vorstellungen von Öffentlichkeit und Sozialität» und als dialektische Beziehung von «Zeigen und Verbergen» (259ff, 276). Neue Formen der Inszenierung von persönlichen Eigenschaften und Intimität verweisen auf neue Formen von Vergesellschaftung, nicht auf deren Verlust, wie Schwietring plausibel argumentiert. Das Verständnis von Gemeinschaft als Individualität und Intimität ausschließende Beziehungsform (vgl. 275) dürfte hingegen im englischsprachigen Raum nicht nur auf Unverständnis treffen (vgl. Joas 2002, 1993), sondern ist empirisch höchst fragwürdig.

Matthias Hoffmann macht in seinem Beitrag die Vorder- und Hinterbühnenmetaphorik von Goffman für die Betrachtung hospizieller Sterbebegleitung fruchtbar (549-571). Die geforderte Natürlichkeit des Da-Seins im Umgang mit den Sterbenden ist eine künstliche, die überwiegend über die Hinterbühne erzeugt wird (vgl. 552, 569).

\section{Theatralität im Kontext von Wissen und Reflexion}

Mit einem etwas anderen Zeigen, nämlich dem von Wissen in Präsentationen beschäftigt sich Hubert Knoblauch in seinem Beitrag (vgl. 221ff, insbesondere 234). Wissen wird im Zeigen räumlich-performativ, wobei die «Pseudospontaneität» des Hybridmediums Präsentation als Medium der Wissensgesellschaft (236) erst den performativen Charakter erzeugt, der Wissen sichtbar macht.

Udo Thiedeke beschreibt in seinem Beitrag «Mode als paradoxes Reflexionsmedium» (183) jene als performative Konstitution situativer normativer Ordnung. Durch ihre schwache Kraft zur Formgebung ist Mode besonders in komplexen (pluralistischen) Gesellschaften attraktiv (vgl. 199), die durch sie konstituierte Gemeinsamkeit liegt darin, anders sein zu wollen. Ihre paradoxe Reflexivität bezeichnet die gesellschaftliche «Inklusion des Abweichenden durch Selbstbeobachtung sozialer Attraktivität» (199). 


\section{Theatralität von Gemeinschaft}

Der an sehr verschiedene Theorietraditionen anknüpfende Beitrag von Gallina Tasheva beschäftigt sich rationalitätskritisch mit der Theatralität des Todes und mit Opferriten. Ritualtheoretisch anknüpfend an die Vorstellung von unbewusster (funktionaler) Theatralität zeichnet die Autorin die kulturelle Bedeutung des (theatralen) Umgangs mit dem ritualisierten Tod als «Zugang zum originär Unzugänglichen» $(289,295)$ und er-/gefundener Verweis auf das «Dasein als das schlichtweg Undarstellbare» (297).

Die Theatralität von Gemeinschaften wird auch im Beitrag von Thorolf Lipp behandelt, der das Turmspringen von Pazifikinsulanern als «riskantes Spektakel» (325) und tendenziell «degeneriertes Ritual» (326) beschreibt, das als universelle Form des sozialen Dramas Parallelen zu modernen Gesellschaften aufweist und (gemeinschaftskonstitutive) Grenzerfahrungen ermöglicht.

Marèn Schorch beschreibt in ihrem Beitrag sehr anschaulich das sorbische Osterreiten als rituelle und symbolische Inszenierung von Zugehörigkeit zu einer (sprachbezogenen und katholisch geprägten) Minderheit (vgl. 331ff) und als «Zeugnis von Reethnisierung» (350ff).

Die Grenzen des Theatermodells reflektieren Rainer Diaz-Bone als auch Ronald Hitzler und Michaela Pfadenhauer in ihren Beiträgen. So kritisiert Diaz-Bone am Beispiel der «Tangowelt Berlin» (355-376) das zunehmend entleerte Konzept der Performanz und dessen Ausblendung vorausgehender Strukturierungen und (reflexiver) diskursiver Ressourcen des Feldes als auch die vereinseitigende Betonung von Situativität und kreativer Praxis. Hitzler und Pfadenhauer hinterfragen im Kontext der diversifizierten TechnoPraxis (377-392) die Vorstellung von einem Publikum, die das Selbstverständnis der Akteure nicht trifft (vgl. 390f)

Methodisch problematisieren Claudia Bullerjahn und Stefanie Heipcke in ihrem Beitrag über Karaoke als «Phänomen junger Unterhaltungskultur» (414) situativer Gemeinschaften das Interview als für Aspekte nonverbaler Selbstdarstellung eher untaugliches Erhebungsinstrument (vgl. 415). 


\section{Rezensionen}

\section{Verwertungsbezogene Theatralität}

\section{Theatrale Kommerzialisierung}

Jürgen Schwier und Thorsten Schauerte beschreiben in ihrem Beitrag zur "Theatralisierung des Sports» (419-438) die Anpassung von Sportarten an medienökonomische Bedingungen, wobei die Unterhaltungsorientierung des Mediensports auch Sportarten erschafft, die die Genregrenzen von Sport und Unterhaltung transzendieren, wie beispielsweise das Wrestling (vgl. 432ff). Hans Ulrich Gumbrecht beschreibt die Theatralisierung des Sports als dessen Sakralisierung: Sport wird als außeraltägliches Reich der Wunder zur gemeinschaftsstiftenden Quasi-Religion, die tiefe Dankbarkeit hervorruft und einen transzendentalen Horizont markiert (vgl. 445).

Die Inszenierung des Shoppings beschäftig Kai-Uwe Hellmann in seinem Beitrag, wobei er zwischen Zweckeinkauf und Erlebniseinkauf unterscheidet (vgl. 585, 588) und für letzteres als strategischer Verschmelzung von Einkauf und Theater anhand der Theatermetaphorik Rollenteilungen, Inszenierungsaufwand und -wirkung, Aufführung von Möglichkeitswelten usw. thematisiert (vgl. 587, 589). Die Gültigkeit dieser Metaphorik sieht er jedoch nicht mehr außerhalb von face-to-face-Interaktionen, etwa für Formen des eCommerce gegeben (vgl. 592).

\section{Theatrale Distinktion}

Die Selbstinszenierung von akademischen Akteuren und Hochschulen wird in den Beiträgen von Dietrich Schwanitz († 2004), Justine Suchanek als auch im Kontext der Inszenierung von wissenschaftlicher Exzellenz durch Richard Münch aufgegriffen. Schwanitz beschreibt eindrucksvoll verschiedene Plattformen der karrierebezogenen Selbstdarstellung von Akademikern und ordnet diesen Bühnen (bspw. Kongresse, Publikationen, Paradigmenkämpfe, Gremiensitzungen), Verhaltensmuster und Rollen wie den Imponiertyp, den Entertainer und den Polemiker zu (vgl. 453ff). Fraglich bleibt trotz treffender Beschreibung akademischer Selbstdarstellungsformen die Lokalisierung von Artikulationstabus in der 68er-Kulturtradition. Zu vermuten ist, dass sich jene Tabuisierung aus sehr verschiedenen Quellen speist, etwa auch aus der Theatralität politisch induzierter, akademischer Leistungsideologie. 
Die Problematik jener politisch inszenierten Leistungsideologie für akademische (Selbst-)Darstellungspraxis wird im Beitrag von Richard Münch (485497) deutlich, der die expertokratische Scheinobjektivität des politisch inszenierten Wettbewerbs um Forschungsförderung als wissenschaftlich illegitime Konstruktion von Exzellenz und «Spitzenuniversitäten» problematisiert (vgl. 489, 495). Der Exzellenz-Rhetorik und damit verbundenen Selbstdarstellungs- und Legitimationspraxen liegt ein Rationalitätsmythos zu Grunde, welcher verschleiert, dass Exzellenz aus kartellartigen Strukturen in Diskrepanz zum faktischen wissenschaftlichen Erfolg (bspw. Publikationsoutput, Zitationen, Patente, vgl. 492) konstruiert wird (vgl. 493).

Justine Suchanek zeigt anhand einer inhaltsanalytischen Auswertung von Hochschulleitbildern auf Internetpräsenzen von ausgewählten Universitäten und Fachhochschulen der Bundesrepublik konvergente und überstilisierende Selbstdarstellungspraxen als Reaktion auf Globalisierungsanforderungen (vgl. 469ff, 475f). Hierzu gehören die semantische Überstilisierung der Hochschulen als interdisziplinäre Forschungsorganisationen jenseits tatsächlicher Forschungspraxis (vgl. 478), die semantische Übersteigerung der Leistungen für die Gesellschaft (vgl. hier wäre zu reflektieren, dass diese artikulierten Leistungen innerhalb von kulturellen Diskursen situiert sind, etwa in der hier beschriebenen zweckrationalen Verkürzung), die wirklichkeitsferne Selbstdarstellung als unternehmerische Hochschule und eine Überstilisierung der eigenen Attraktivität für ausländische Studierende (vgl. 479f).

Anhand von Stelleninseraten in der FAZ (Frankfurter Allgemeine Zeitung) konstatieren Justine Suchanek und Barbara Hölscher einen verstärkten Rückgriff auf nicht zertifizierbare «Attribute und Qualitäten der Person und der Persönlichkeit» (611). Neben dem gewachsenen Bedarf nach Vernetzungswissen wird hier die Fähigkeit zu Selbstmanagement eingefordert, deren Nachweis der «Kompetenzdarstellungskompetenz» des Bewerbers obliegt (612). 
Cornelia Koppetsch analysiert Sinnkonstitution und Legitimation von sozialer Ungleichheit am Beispiel der Kultur- und Medienberufe als Avantgarde des Kulturwandels von Arbeit, in welcher das «Ethos der Kreativen zum Subjektund Berufsideal geworden ist» ${ }^{1}$ (575). Im «Berufshabitus der Werbegestalter» wurde das Ideal kreativer Arbeit mit den «wettbewerbsorientierten Arbeitswelten des globalen Kapitalismus» versöhnt 579), so dass Wettbewerbe zu legitimen Wertigkeitsprüfung avancieren (vgl. ebd.).

\section{Theatrale Gewalt}

Bernhard Giesen beschreibt Terrorakte als «theatralische Darbietungen, die gezielt für ein Publikum gemacht sind.» (616). Ihn interessieren die Mythen, Skripte und Narrative, derer sich der Terrorismus in seiner dichotomen Weltbetrachtung bedient (vgl. 73) und die in kulturspezifischen Heldenidealen (vgl. a.a.O.: 616) als auch kulturellen Befreiungsmythen und -erzählungen zu finden sind (vgl. 618f). Terror ist zudem Aufführung für ein zweigeteiltes Publikum: Befürworter und Unverständige (vgl. 619).

\section{Theater und (Ent-)Theatralisierung}

Um die (Ent-)Theatralisierung des Theaters geht es in den Beiträgen von Rüdiger Lautmann und Erika Fischer-Lichte. Während Lautmann auf Basis von Zuschauerbeobachtung und Presseberichterstattung von einer zunehmenden Theatralisierung des Theaters etwa durch abnehmende Stücktreue, gestiegene Individualität der Darsteller und Selbstreflexivität infolge gestiegener Differenzierung ausgeht (vgl. 505ff), konstatiert Fischer-Lichte anhand ausgewählter Stücke eine Enttheatralisierung des Theaters im Gefolge einer Theatralisierung des öffentlichen Lebens, die sie als problematische Entwirklichung versteht (vgl. 531f).

In seinem Beitrag über die «Theatralisierung des Therapeutischen» markiert Matthias Warstat die strukturellen Differenzen zwischen Theatertherapie und Kunsttheater und betont den Stellenwert von «Verkörperung und Projektion, Einführung und Distanzierung» im Probenprozess für Selbstreflexivität (vgl. a.a.O.: 71). Während das Kunsttheater vom Individuellen auf öffentliche Fragen und Probleme reflektiert, fokussiert das Therapietheater über assoziierte Gruppen individuelle Strategien und Lösungen (vgl. 546).

\footnotetext{
${ }^{1}$ Diese Perspektive wurde insbesondere durch die Diagnose der digitalen Boheme über Fachdiskurse hinaus belebt, was im Beitrag ausgeblendet bleibt (vgl. Friebe 2006).
} 


\section{Medientheatralität und Medientheatralisierung (Band 2)}

Im zweiten Band sind Beiträge versammelt, die sich schwerpunktmäßig mit Medien-Theatralität im Kontext von Massenmedien und massenmedialen Programmbereichen wie auch dem Internet beschäftigen. Diese unterscheidet sich von «Interaktionstheatralität» (womit face-to-face-Interaktionen gemeint sind) dadurch, dass die Ebenen und Komponenten von Theatralität wie Performance, Inszenierung, Korporalität und Wahrnehmung anders strukturiert sind (vgl. Willemsen, Bd. 1: 89ff). Der zweite Band enthält neben einer Einleitung des Herausgebers und der Zusammenfassung der Einzelbeiträge drei Hauptkapitel, die mit «Massenmedien», «Internet» und «Globale Diagnosen» betitelt sind.

Willems konzeptualisiert in seiner Einleitung zum zweiten Band den soziologischen Begriff der Medientheatralität in Rekurs auf verschiedene soziologische Ansätze wie die Figurationssoziologie, die Wissenssoziologie, die Diskurstheorie und die Systemtheorie (vgl. 15ff). Das Modell des Theaters soll Perspektiven auf die Wirklichkeit eröffnen, die die "(Medien-)Theatralität als solche darstellt» und auf jene, die durch (Medien-)Theatralität hervorgebracht wird (40).

Im zweiten Band dominiert (sicher nicht zufällig) die Auseinandersetzung mit der mediatisierten Theatralisierung von Werbung und Vermarktung.

Daneben finden sich Beiträge über die Theatralisierung des Politischen, des Religiösen, der Privatheit, der Intimität und des Selbst, des Sports und der Ökonomie im Allgemeinen.

\section{Theatralität mediatisierter Politik}

Christian Schicha veranschaulicht in seinem Beitrag anhand der Theatralität der massenmedialen Debatte zum Zuwanderungsgesetz 2002 die Fruchtbarkeit des Theatralitätskonzeptes für die Beschreibung (mediatisierter) politischer Prozesse (vgl. 70). Dabei reflektiert er auf die Problematik der massenmedialen Dominanz der Inszenierung über Inhalte in der Theatralität des Politischen (vgl. 61, 64). und zeigt die Abhängigkeit der Dramaturgie politischer Ereignisse und des Diskursniveaus von der jeweiligen Medienlogik (Nachrichtenwert, medienökonomische Kontexte, Formate usw; vgl. $65 \mathrm{ff})$. 
Die Theatralität des Politischen betrachtet Hans Manfred Bock mit Blick auf den französischen Intellektuellen. Prägnant schildert er die Transformation des zeitgenössischen Intellektuellen, die sich neben medienökonomischen Bedingungen auch kulturellen Veränderungsprozessen im Kontext von (Werte-)Pluralisierung und (Bildungs-)Demokratisierung verdankt. Während die (un-)freiwillige Unterwerfung des zeitgenössischen Intellektuellen «unter die medienökonomischen Innovations- und Rehabilitationszwänge» an die Stelle der öffentlichen Intervention qua kultureller Reputation Prominenz «aufgrund medialer Performanz und Selbstinszenierung» setze (88), lösen benannte kulturelle Veränderungen den einstigen Stellvertreter-Status des klassischen Intellektuellen ${ }^{2}$ dahingehend auf, dass der neue Medienintellektuelle im extremsten Falle nichts mehr außer sich selbst vertritt und zwar in leistungsfähigen Medien der Selbstinszenierung (vgl. 95).

Andreas Langenohl und Kerstin Schmidt-Beck hinterfragen «den zeitdiagnostischen Anspruch des Theatralitätstheorems» (114) anhand von Interviewmaterial mit professionellen Akteuren des Finanzmarktes. Hier wird die Ambivalenz eines zunehmend prekärer werdenden Medienverhältnisses zwischen eingeforderter massenmedialer Selbstinszenierung als auch Repräsentation der Organisation und einer finanzkrisenevozierten Riskanz von Medienpräsenz etwa infolge der Personalisierung und Emotionalisierung von Schuld und Verantwortung deutlich (vgl. 111f).

\section{Theatralisierung mediatisierter Religiosität}

Andreas Hepp, Veronika Krönert und Waldemar Vogelgesang setzen sich mit der zunehmenden Mediatisierung des Religiösen am Beispiel des XX. Weltjugendtages auseinander. Die Individualisierung religiöser Teilhabe durch mobile Kommunikationstechnologien (Mobilität, translokale Verbundenheit, mobile religiöse Expressivität; vgl. 128) als auch das Branding von Religion als Medienevent in der Berichterstattung sind empirische Indikatoren mediatisierte Religion und Religiosität, die auch die katholische Kirche dazu zwingt, «ihr Glaubensangebot medial angemessen zu inszenieren» (134). Mit anderen Worten, Theatralisierung ist als ein Aspekt von Mediatisierungsprozessen anzusehen.

${ }^{2}$ Wenngleich auch der klassische Intellektuelle Tendenzen einer Selbststilisierung aufwies (vgl. hierzu Habermas 2006). 


\section{Theatralität von Fernsehformaten}

Dies wird auch in dem Beitrag von Axel Schmidt, Andrea Teuscher und Klaus Neumann-Braun deutlich, die in ihrem Beitrag die Inszenierung von Face-to-Face-Interaktion beschreiben. Am «prototypischen» Beispiel des Gästetalks der Sendung TV-Total mit Stefan Raab thematisieren sie die gelungene Aufführung von Vorführung (vgl. 266ff). Diese mediale Theatralitätskultur operiert als (wirklich nur scheinbare?) Entlarvung problematischer Medienpraxen mit der (ungleich verteilten) Möglichkeit des Gesichtsverlustes im Rahmen des Dokutainment-Fernsehens und des Duell-Formats (vgl. 289), wobei der Gesprächspartner zum «Sparringpartner» wird.

Auch im zweiten Band findet sich ein Beitrag über Fernsehsport. Lothar Mikos konstatiert exemplarisch anhand des Fußballs, dass Professionalisierung und Kommerzialisierung eine symbiotische Beziehung zwischen Sport und Medien hervorgebracht haben, die zwei Wirklichkeiten produzierte, die es Sports und die des Fernsehsports. Letztere präsentiere Sportereignisse als mehrfach gerahmte theatrale Aufführungen, etwa in der medialen Konstruktion von Stars und (tragischen) Helden, die kulturspezifische Werte, Normen und Rollenbilder verkörpern (vgl. 42, 145ff, 153).

Ein zweiter, sehr interessanter Beitrag von Lothar Mikos beschäftigt sich mit dem Fernsehformat Daily Talks als Theatralisierung von Privatheit. Hier konstatiert er, dass dieses Fernsehformat, welches durch einen «Diskurs der Betroffenheit» (224), durch Selbstdarstellung und die Inszenierung von Privatheit und Alltag charakterisiert ist, die fortgeschrittene Mediatisierung der Alltags- und Lebenswelt aufzeigt (vgl. 240). Hier (und mittlerweile in einem erweiterten Formatrepertoire) würde in charakteristischer Personalisierung und Emotionalisierung der moralische Konsens der Gesellschaft entlang abweichenden Verhaltens und abweichender Lebenssituationen und damit verknüpfte Wert- und Normbezüge verhandelt (vgl. 241). In diesem Zusammenhang stellt sich die Frage, inwiefern jene mediatisierte (formatspezifische) Theatralisierung von Moral Reflexionsräume eröffnet oder aufgrund des inszenierten Involvements (eben jener Diskurs der Betroffenheit) eher begrenzt (vgl. Marotzki 2007). 
Auch Jo Reichertz setzt sich in seinem Beitrag mit folgenreicher Theatralität und deren impliziten Wert- und Normbezügen auseinander. Reichertz rekonstruiert Liebesshows im Fernsehen und attestiert der (öffentlichen) Darstellung von Liebe zunehmende Visualisierung und Überführung von Worten in Handlungen (vgl. 258f), wobei theatrale Liebesdarstellungen auf die «Macht der anwesenden Zuschauer und Zeugen» setzen (259). Für inn ist das ein Hinweis auf die Verschiebung von Liebesidealen: Liebe bemisst sich (milieuspezifisch ist dem ggf. hinzuzufügen) nicht mehr nur in der Tiefe individueller Gefühle für den anderen, sondern darin, was «Liebende bereit sind, füreinander unter den Augen der Öffentlichkeit zu tun» (ebd.).

\section{Theatralität von Werbung}

Der erste Beitrag im Cluster Theatralität von Werbung ist von Ralf Vollbrecht und beschäftigt sich mit dem (funktionalen) Wandel der theatralen Konstellation der Werbung. Neben neuen Inszenierungsmodi wie der dekonstruierenden oder selbstironisierenden Werbung, individualisierter (Online-)Werbung und Schockwerbung ist dieser Wandel vor allem durch neue Formen des «Mitmach-Theaters» als ko-evaluativer Mitgestaltung von Werbung im Kontext von Online-Communities charakterisiert (vgl. 42f, 164f).

Der Wandel von Werbung steht auch im Zentrum des Beitrages von Michael Jäckel und Cornelia Eck. Anhand einer (triangulierenden) Anzeigenanalyse fokussieren sie den in Werbung aufgeführten Wandel von Geschlechterrollenverständnissen (vgl. 173ff, 182). Fraglich ist, inwieweit der Wandel von Geschlechtsrolleneinstellungen lediglich eine Erweiterung des männlichen Rollenkonzeptes darstellt (vgl. 182).

Methodisch anders gelagert ist der Folgebeitrag, an dem neben Julia M. Derra ebenfalls Jäckl beteiligt ist. Hier werden Inhaltsanalysen mit einer Rezipientenanalyse verknüpft, um geschlechtsspezifische Identifikationsgrade bzw. die Akzeptanz von geschlechtsspezifischen Aufführungen von FrauSein in der Werbung zu eruieren (vgl. 206).

Dietrich Schwanitz († 2004) widmet sich im zweiten Band der Werbung für Literatur und dem Bestseller-System aus der Perspektive des teilnehmenden Beobachters und Hobby-Soziologen (vgl. 211ff). In bekannter Manier karikaturiert er hier die Theatralisierung von Literaturwerbung über die (Selbst-)Inszenierung des Produktes und des Produzenten (bspw. Starautoren) in verschiedenen medialen Plattformen, was sogar dazu führt, dass die 
Werbung der Herstellung des Produktes vorausgeht (vgl. 215) oder das Produkt Beigabe zum verkauften Lebensstil wird (vgl. 218).

Aus der Perspektive des Markenmanagements betrachten Sven Henkel und Benjamin von Walther theatrale Aspekte der «Marke Mensch» (312) und die damit verbundenen Bedingungen von Markenloyalität, wobei sie die Relevanz der Medien-Bühne und ihrer Eigenlogik (Formatlogik) für die Inszenierungsoptionen der Marke herausstellen (vgl. 323).

Die kulturelle Bedeutung der Theatralisierung von Persönlichkeiten betrachtet Katrin Keller in ihrem Beitrag aus identitätstheoretischer Perspektive. Sie bezeichnet Stars als «personale parasoziale Identitäts-Anker» (46), die insbesondere von ihrer imaginierten und inszenierten Privatheit her auf die «Nutzer» wirken.

Udo Göttlich und Jörg-Uwe Nieland beschreiben Erscheinungsformen des Kult-Marketing, die mit einer neuen Form von situativen Stars und Starkarrieren einhergehen (vgl. 305). Die langsame Ausweitung des KultMarketings in Eliten und Szenen neben der Ausrichtung auf Populärkultur bezeichnet die Aufmerksamkeitsökonomie der «Kultur des mentalen Kapitalismus» (306), in welcher der öffentliche Raum in eine riesige Werbefläche für Produkte jedweder Art verwandelt wird.

Die folgenden Beiträge zum Themenfeld «Internet» beinhalten ebenfalls zwei Beiträge, die sich mit der Theatralität von Werbung beschäftigen.

Caja Thimm beschreibt neue Werbeformen im Internet, etwa im Kontext von E-Mails, Interstitials, PopUps, Bannern und Buttons als auch hinsichtlich partizipativer community-bezogener Werbung (vgl. 401ff). Die Medienspezifik internetbasierter Werbung liegt besonders auf der Generierung aktiver Aufmerksamkeit (vgl. 412).

Auch Eva Lia Wyss beschäftigt sich mit Werbung im Internet (als auch dem Fernsehen und in Zeitungen), wobei sie das ästhetisierende Verstecken des Werbenden der Werbung fokussiert (vgl. 49, 417ff). Dieses neue Paradigma versteht sie als einverleibenden Metatext. In Bezug auf das Internet werden tatsächlich versteckende Werbeinszenierungen von offenkundig versteckender Werbung unterschieden und problematisiert (vgl. 427). 


\section{Theatrale Strukturen des Internets}

Mike Sandbothe entwickelt in seinem Beitrag zeichentheoretisch eine Phänomenologie theatraler Merkmale des Internets, die er auch als neue Verflechtungsprinzipien in der semiotischen Tiefentheatralisierung des Internets beschreibt. Dazu gehören die Verschriftlichung der Sprache als performatives Schreiben eines Gesprächs (bspw. Chat), die Verbildlichung der Schrift («bildhafte Dramatisierung des Zeichenarrangements», 359) und die Verschriftlichung des Bildes (vgl. 351ff, 357).

Ein anders gelagertes Verständnis von Theatralität verfolgt Sebastian Pranz in seinen Betrachtungen internetspezifischer Theatralität abwesender Körper am Beispiel des Cybersex-Chats (vgl. 367ff, 377), dessen Theatralität für die Akteure immer wieder zum Gegenstand bewusster Reflexion wird (vgl. 381). Theatralität wird hier an Rahmenwissen gebunden und als «höchst voraussetzungsvolle Interpretationsleistung des Zuschauers» verstanden (382).

Klaus Neumann-Braun beschäftigt sich in seinem Beitrag mit verschiedenen Formen inszenierter Selbstpräsentation im Internet: den Webcam-Auftritten der Altäglichkeit von «jedermann» und den ko-evaluierten Auftritten Jugendlicher auf der Videoplattform youtube (vgl. 390ff). Während im ersteren Aufführungsfall Authentizität als relevantes Bewertungskriterium betont wird, geht es im letzteren Fall um die intersubjektive Legitimität identitätsrelevanter Identifikationen.

\section{Theatralisierungsmetaphorik und gesellschaftliche Entwicklung}

Das abschließende Kapitel des zweiten Bandes bilden die Beiträge von Peter Ludes und Cornelia Klinger, die allgemeinere Entwicklungstendenzen mediatisierter Theatralisierung im Kontext von Zivilisationstheorie (Ludes) und Polarisierungsphänomenen (Klinger) konturieren.

Vor dem Hintergrund des formulierten Anspruches, einen integrativen Blickwinkel auf (Ent-)Theatralisierungsphänomene zu entwickeln, wäre eine stärkere Vermittlung der Einzelbeiträge wünschbar, wenngleich aufgrund der Heterogenität der Forschungsansätze nur schwer realisierbar. Eine Möglichkeit hierzu hätte ein synthetisierendes Schlusswort bieten können. 
Nicht zuletzt greifen der Herausgeber und die Autoren der beiden Sammelbände Phänomene sozialen Wandels auf, die in der Tat einen umfassenden Veränderungsprozess hin zu einem dramaturgischen Bewusstsein beschreiben (vgl. Rifkin 2009, 558ff), der mit Mediatisierungsprozessen (vgl. Lundby 2009) und mit dem als Wertewandel beschriebenen Veränderungen der Einstellungen und des Verhaltens in engem Zusammenhang steht. Insgesamt bieten die beiden Bände eine breite, teilweise sehr anregende Sicht auf Phänomene jenes entstandenen dramaturgischen Bewusstseins und sind zu empfehlen.

Daniela Küllertz

\section{Literatur}

Friebe, Holger; Lobo, Sascha. Wir nennen es Arbeit: Die digitale Boheme oder: Intelligentes Leben jenseits der Festanstellung. München: Heyne Verlag, 2006.

Habermas, Jürgen. Ein avantgardistischer Spürsinn für Relevanzen. Was den Intellektuellen auszeichnet. Dankesrede von Jürgen Habermas anlässlich der Verleihung des Bruno-Kreisky-Preises für das politische Buch 2005. Verfügbar über: http://www.rennerinstitut.at/download/texte/habermas2006-03-09.pdf (Stand 15.02.2008), 2006.

Joas, Hans. Ungleichheit in der Bürgergesellschaft. Über einige Dilemmata des Gemeinsinns (Vorlesung an der Berlin-Brandenburgischen Akademie der Wissenschaften am 14. Dezember 2000). In: BerlinBrandenburgische Akademie der Wissenschaften: Berichte und Abhandlungen, Bd. 9, Berlin: Akademie-Verlag, 2002.

Joas, Hans. Gemeinschaft und Demokratie in den USA. Die vergessene Vorgeschichte der Kommunitarismus-Diskussion. In: Brumlik, Micha / Brunkhorst, Hauke (Hrsg.), Gemeinschaft und Gerechtigkeit. Frankfurt: Fischer, 1993, S. 49-62.

Lundby, Knut (Hg.). Mediatization: Concept, Changes, Consequences. New York: Peter Lang, 2009.

Marotzki, Winfried. Die Macht der Erinnerung - Involvement und Reflexion. Aspekte einer strukturalen Medienbildung am Beispiel Film. In: Fromme, Johannes / Schäffer, Burkhard (Hg.): Medien - Macht - Gesellschaft. Wiesbaden: VS Verlag, 2007.

Rifkin, Jeremy. The Empathic Civilization. The Race to Global Consciousness in a World in Crisis. New York: Penguin Group, 2009. 